\title{
Magnetically modulated optical transmission of magnetic fluid films
}

\author{
H.E. Horng ${ }^{\mathrm{a}, *}$, S.Y. Yang ${ }^{\mathrm{b}}$, W.S. Tse ${ }^{\mathrm{b}}$, H.C. Yang ${ }^{\mathrm{c}}$, Weili Luo ${ }^{\mathrm{d}}$, \\ Chin-Yih Hong ${ }^{\mathrm{e}}$ \\ a Department of Physics, National Taiwan Normal University, sec 4, Taipei 116, Taiwan \\ ${ }^{\mathrm{b}}$ Institute of Physics, Academia Sinica, Taipei 115, Taiwan \\ ${ }^{\mathrm{c}}$ Department of Physics, National Taiwan University, Taipei 106, Taiwan \\ ${ }^{\mathrm{d}}$ Department of Physics, University of Central Florida, Orlando, FL 32816, USA \\ ${ }^{\mathrm{e}}$ Department of Mechanical Engineering, Da-Yeh University, Chang-Hwa 515, Taiwan
}

\begin{abstract}
The optical transmission of the magnetic fluid film under perpendicular magnetic fields was investigated by measuring the transmitted intensity of the normally incident light through the film. The transmittance $I / I_{0}$ of the magnetic fluid film was observed to decrease with the increase of the magnetic field strength $H$ due to the reduction in the area occupied by the relatively optic transparent liquid phase. Moreover, under a given $H$, the transmittance decreased exponentially with the increasing film thickness $L$, i.e. $I / I_{0}=\exp (-L / \delta)$, where the $\delta$ denotes the attenuation distance. It was further found that the $\delta$ varies with the magnetic field strength and the concentration of the fluid, but is independent of the sweep rate of the field.
\end{abstract}

(C) 2002 Elsevier Science B.V. All rights reserved.

Keywords: Magnetic fluid; Transmittance; Attenuation distance

\section{Introduction}

Magneto-optics of magnetic fluids has recently attracted sustained interests due to the richness of this relatively unexplored phenomena. Within the research area, the study of optical transmittance of magnetic fluid films under external magnetic fields is growing all over the world because of the feasibility of optical devices such as the optical switch [1], the magnetic field sensor [2] and modulators [3] etc. It is indicated that the transmittance of the magnetic fluid film can be manipulated by adjusting the magnetic field strength $[1,4]$. In addition, the temperature [5] and wavelength effect [6] on the transmittance were also reported. The physical mechanism accounting for the variation in the resultant transmittance is indicated to be directly

\footnotetext{
*Corresponding author. Tel.: + 886-2-8931-6233; fax: + 8862-2930-9439.

E-mail address: phyfv001@scc.ntnu.edu.tw (H.E. Horng).
}

attributed to the evolution of the structure in the magnetic fluid film under magnetic fields [2]. By taking account of the variations of the effective concentration and the area ratio of the liquid phase in the magnetic fluid film under magnetic fields, the resultant magnetic field dependence of the optical transmission can be predicted [2].

In our previous studies $[7,8]$, it was found that the structural patterns could be affected by the control parameters, such as the field strength, the sweep rate of the field, the film thickness and the concentration of the magnetic fluid. This implies that the transmittance of the magnetic fluid film should also be determined by these control parameters. Even today, the relationship between the transmittance of the magnetic fluid film and the control parameters is still not understood well. In this study, we systematically investigate the influences of the film thickness, the concentration, and the sweep rate on the magnetically modulated transmittance of magnetic fluid films. 


\section{Experimental details}

The magnetic fluid was prepared with the chemical coprecipitation technique. The magnetic particle of the ferrofluid is $\mathrm{Fe}_{3} \mathrm{O}_{4}$ with a size around $118 \AA[9,10]$. The fluid was then injected into glass cells to form magnetic fluid films. A pair of solenoids was used to provide a magnetic field perpendicular to the plane of the magnetic fluid film. Throughout the experiment, the temperature was kept at $24.3^{\circ} \mathrm{C}$. To measure the optical transmission of the magnetic fluid, an unpolarized parallel light ray with a wavelength of $1557 \mathrm{~nm}$ was perpendicularly incident to the film, and the transmitted light was guided, by an optical fiber, to the spectroscopy to measure the transmitted intensity. By dividing the measured transmitted intensity $I$ of the magnetic fluid film under a given field by that, $I_{0}$, of the bare glass cell, the transmission of the film was obtained. The structures of the magnetic fluid film under fields were observed with an optical microscope and then photographed with a CCD camera.

\section{Results and discussion}

Due to the formation of columns agglomerated by magnetic particles, the optical transmittance of the film can be modulated by external magnetic fields [2]. For the magnetic fluid film of a given thickness, the transmittance $I / I_{0}$ of the film was observed to decrease with the increasing magnetic field $H$. A typical $I / I_{0}-H$ curve of the magnetic fluid film is shown in Fig. 1 . As the film thickness $L$ was increased, the $I / I_{0}-H$ curve moved to the region of lower transmittance. This movement in the $I / I_{0}-H$ curve is attributed to the elongation of the traveling path as the light passes the films. The inset in Fig. 1 plots the film-thickness dependence of transmittance under various $H$ 's in the log-linear scale. It was found that the transmittance follows the relation $I / I_{0}=$ $\exp (-L / \delta)$, where $\delta$ denotes optical attenuation distance and the value of the $-1 / \delta$ for a fixed $H$ was the product of the $\ln 10$ and the slope of the corresponding $\log \left(I / I_{0}\right)$ $L$ curve shown in the inset. According to the experimental data, the various $\delta$ 's resulted under various $H$ 's. Here, the $\delta$ ranged from 80 to $50 \mu \mathrm{m}$ as the $H$ increased from zero to $300 \mathrm{Oe}$.

In addition to the magnetic field and the film thickness, the transmittance of the magnetic fluid film was also observed to be dependent on the concentration of the fluid because of the enhancement in the optical scattering as light passes through the film of a higher concentration. This implies that the attenuation distance $\delta$ should be reduced for a magnetic fluid film of higher concentration. To check this, the $\log \left(I / I_{0}\right)-L$ curves were measured for the magnetic fluid films of various concentrations under either zero or nonzero magnetic

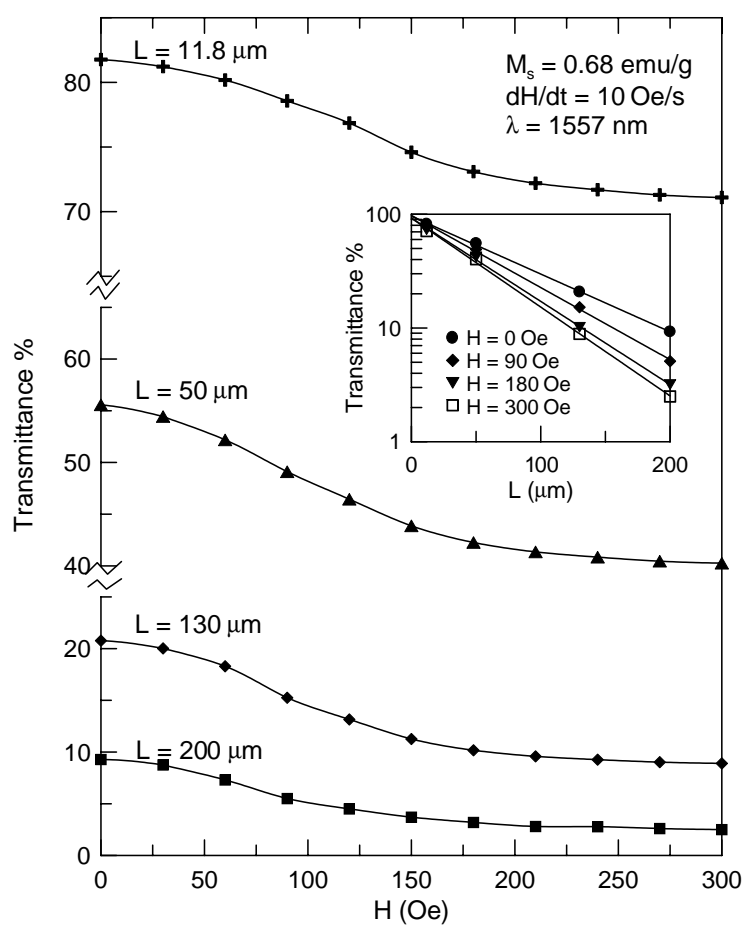

Fig. 1. Transmittance versus magnetic field strength for various film thickness $L$ 's. The inset shown the film-thickness dependent transmission of the magnetic fluid film in log-linear scale.

fields. For the zero field, it was found that the slope, $s$, of the $\log \left(I / I_{0}\right)-L$ curve changes with the concentration of the fluid, as shown in Fig. 2(a). Thus, the attenuation distance $\delta$ is indeed a function of the fluid concentration via the relation $\delta=-1 /(s \ln 10)$. Through a careful analysis of the results in Fig. 2(a), the $\delta$ was found to decrease from 279 to $38 \mu \mathrm{m}$ as the concentration increased from $0.17 \mathrm{emu} / \mathrm{g}(=0.30 \%$ in volume fraction $)$ to $1.08 \mathrm{emu} / \mathrm{g}(=1.93 \%)$. This phenomenon was also observed for a nonzero field, say $120 \mathrm{Oe}$, as shown in Fig. 2(b). It is worth noting that the $\log \left(I / I_{0}\right)-L$ curve under $120 \mathrm{Oe}$ is steeper than the corresponding curve under zero field for a given concentration. Hence, a smaller value is for the $\delta$ under a higher field strength. This result is consistent with that shown in the inset of Fig. 1.

Finally, the influence of the field sweep rate on the magnetically modulated transmission of the magnetic fluid films was investigated. The $I / I_{0}-H$ curves for magnetic fluid films with $0.17 \mathrm{emu} / \mathrm{g}$ of 130 and $200 \mu \mathrm{m}$ in thick were probed at various $\mathrm{d} H / \mathrm{d} t$ 's and are shown in Figs. 3(a) and (b), respectively. It is clear that the $I / I_{0}-H$ curves for various $\mathrm{d} H / \mathrm{d} t$ 's coincide with each other for a given film. To discover the origin of the phenomena in Fig. 3, the structural patterns were examined. It was observed that the columns are 


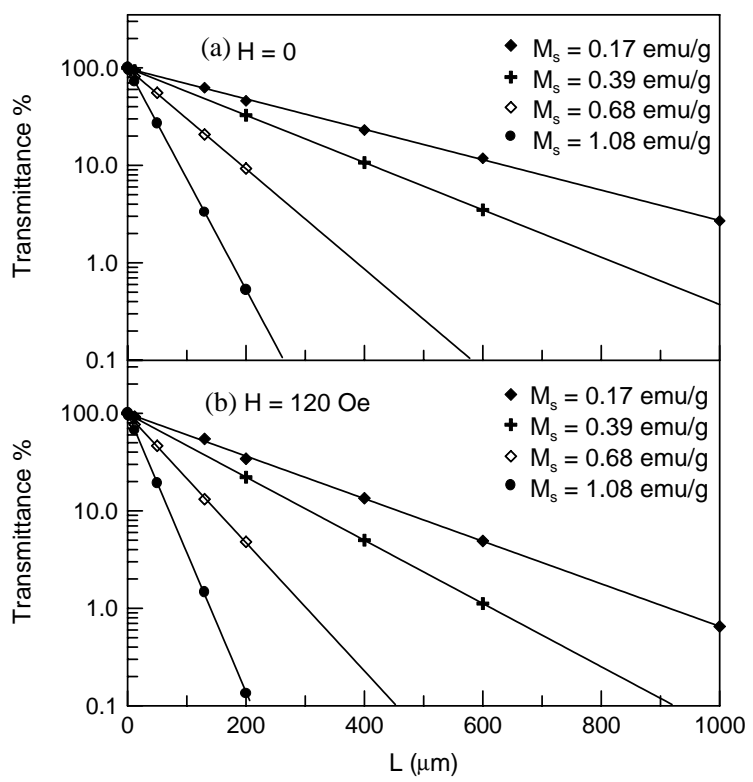

Fig. 2. Transmittance as functions of the thickness of the magnetic fluid films with various concentrations under (a) zero field and (b) 120 Oe.

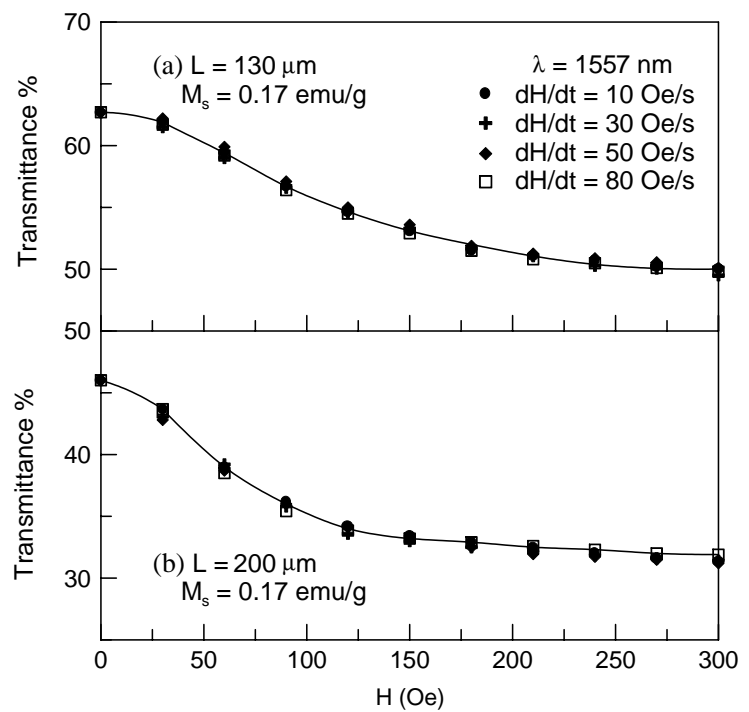

Fig. 3. Magnetic field dependent transmittance of the magnetic fluid films with thickness of (a) $130 \mu \mathrm{m}$ and (b) $200 \mu \mathrm{m}$ at various field sweep rates $\mathrm{d} H / \mathrm{d} t$.

distributed randomly all over the film even $H$ up to 300 Oe. Furthermore, the $\left(A-A_{\mathrm{col}}\right) / A-H$ curves of a film at these $\mathrm{d} H / \mathrm{d} t$ 's were also found to overlap, where $A_{\text {col }}$ denotes the total area of the cross section of the columns within a certain area $A$ of the magnetic fluid film. Thus, under a given $H$, a fixed area was achieved for the optically transparent liquid phase in the film at various $\mathrm{d} H / \mathrm{d} t$ 's. This led to a coincidence of the $I / I_{0}-H$ curves of the magnetic fluid film at various $\mathrm{d} H / \mathrm{d} t$ 's. The inference was obviously consistent with the experimental results shown in Fig. 3. On the other hand, the overlap of the $I / I_{0}-H$ curves for the various $\mathrm{d} H / \mathrm{d} t$ 's implies that the attenuation distance $\delta$ is independent of the sweep rate.

\section{Conclusion}

The transmittance of the magnetic fluid films can be reduced by increasing the magnetic field strength, the film thickness and the concentration of the fluid, but is independent of the field sweep rate. Also, the optical attenuation distance of the magnetic fluid film can be manipulated via varying the field strength and the concentration. Further investigations reveal that the structural patterns are dominant to the transmittance of the magnetic fluid films.

\section{Acknowledgements}

This work is supported by the National Science Council of ROC under grant Nos. NSC89-2112-M-003040 and NSC89-2212-E-212-021.

\section{References}

[1] Chin-Yih Hong, J. Magn. Magn. Mater. 201(1999) 178.

[2] Herng-Er Horng, S.Y. Yang, S.L. Lee, Chin-Yih Hong, H.C. Yang, Appl. Phys. Lett. 79 (2001) 350.

[3] J.-W. Seo, S.J. Park, K.O. Jang, J. Appl. Phys. 85 (1999) 5956.

[4] J.E. Martin, K.M. Hill, C.P. Tigges, Phys. Rev. E 59 (1999) 5676.

[5] Tengda Du, Weili Luo, Appl. Phys. Lett. 72 (1998) 272.

[6] N. Inaba, H. Miyajima, H. Takahashi, S. Taketomi, S. Chikazumi, IEEE Trans. Magn. 25 (1989) 3866.

[7] Chin-Yih Hong, Herng-Er Horng, F.C. Kuo, S.Y. Yang, H.C. Yang, J.M. Wu, Appl. Phys. Lett. 75 (1999) 2196.

[8] Chin-Yih Hong, C.H. Ho, H.E. Horng, Chun-hui Chen, S.Y. Yang, Y.P. Chiu, H.C. Yang, Magn. Gidro. 35 (1999) 364.

[9] Chin-Yih Hong, I.J. Jang, H.E. Horng, C.J. Hsu, Y.D. Yao, H.C. Yang, J. Appl. Phys. 81 (1997) 4275.

[10] Herng-Er Horng, Chin-Yih Hong, Wai Bong Yeung, Hong-Chang Yang, Appl. Opt. 37 (1998) 2674. 